\title{
Diego Alcorta y la difusión de saberes médicos en Buenos Aires, 1821-1842
}

\author{
Mariano Di Pasquale (*) \\ $\left(^{*}\right) \quad$ Instituto de Estudios Históricos, Universidad Nacional de Tres de Febrero/CONICET (Argentina) \\ mariano.dipasquale@gmail.com
}

Dynamis

[0211-9536] 2014; $34(1): 125-146$

http://dx.doi.org/10.4321/S0211-95362014000100007
Fecha de recepción: 27 de noviembre de 2012

Fecha de aceptación: 18 de julio de 2013

SUMARIO: 1.--Introducción: ¿Qué es la Idéologie? 2.-Los antecedentes: la recepción de la Idéologie y el contexto político porteño 3.-Los saberes fisiológicos situados en la Universidad de Buenos Aires 4.-Diego Alcorta y la apropiación del lenguaje fisiológico. 5.-Diego Alcorta y la construcción de una medicina sensualista, práctica y científica. 6.-Conclusiones.

RESUMEN: Este artículo tiene como objetivo analizar los saberes médicos que impulsa el doctor Diego Alcorta derivados de la tradición francesa, en particular los provenientes de la corriente denominada Ideólogie, en el contexto de la Universidad de Buenos Aires entre 1821-1842. Se analiza el proceso de circulación de dichas enseñanzas sumadas a las de la Escuela de Montpellier adaptadas y situadas en el plano local y su paulatino impacto en el ámbito académico, constituyendo un fenómeno relevante en la incipiente conformación de la profesión médica porteña. Alcorta retoma, en especial, la importancia de la observación como método de diagnóstico y curación en el tratamiento de las enfermedades y, la inauguración, en la enseñanza e investigación médicas, de una orientación empírica que introdujo una actividad práctica específica: la clínica hospitalaria. Asimismo, se examina la transmisión de un lenguaje fisiológico y su alcance en las generaciones posteriores preguntándonos qué ocurrió con estos procedimientos prácticos y la difusión de los nuevos saberes fisiológicos. Para responder a esta pregunta compleja se analizan las tesis presentadas y defendidas en el Departamento de Medicina de la Universidad de Buenos Aires durante la época, las que permiten tanto confirmar la presencia del lenguaje sensualista y fisiológico de las enseñanzas de Alcorta como su impacto dentro del mismo ejercicio de la medicina.

PALABRAS CLAVE: Diego Alcorta, saberes médicos, Idéologie, Buenos Aires, siglo XIX.

KEY WORDS: Diego Alcorta, medical knowledge, Idéologie, Buenos Aires, 19th century. 


\section{Introducción: ¿Qué es la Idéologie?}

El presente artículo tiene como propósito analizar los saberes médicos difundidos por el doctor Diego Alcorta, a fin de identificar las conexiones emergentes entre éste y aquellas nociones derivadas de Destutt de Tracy y Pierre Jean Georges Cabanis, referentes de la corriente francesa denominada Idéologie. En particular, se indaga la incorporación de un conjunto de conocimientos sensualistas y fisiológicos los cuales serán transmitidos a partir de su tarea docente en el ámbito de la Universidad de Buenos Aires entre 1827 y 1842, período delimitado por su aparición pública y posterior fallecimiento.

Aunque estos saberes pueden rastrearse en los inicios de la década de 1820, es a partir de la presencia de Diego Alcorta cuando se advierte un proceso de difusión más amplio y ciertas variaciones en la enseñanza y en el ejercicio de la medicina respecto de la etapa anterior. En este sentido, se intenta demostrar que sus orientaciones jalonan un impulso decisivo en el proceso de profesionalización de la medicina local. En pos de dar cuenta del alcance de estas enseñanzas se consideran una serie de tesis presentadas y defendidas para obtener el grado de doctor en medicina por algunos de sus alumnos y discípulos. Su estudio permite constatar la presencia y la transmisión de estos saberes e intentar analizar los cambios que éstos produjeron en la práctica médica local.

En principio, recordemos que la Idéologie como movimiento intelectual ha tenido un escaso tratamiento en los manuales de historia del pensamiento político y en las historias de las filosofías. Tampoco existen demasiados libros o artículos específicos que se refieran exclusivamente al estudio de esta escuela francesa ${ }^{1}$. Sin embargo, no se busca responder a las

1. Sobre la Idéologie, véanse Picavet, François. Les Idéologues, essai sur I'histoire des idées et des théories scientifiques, philosophiques, religieuses, etc. France depuis 1789. Paris: Félix Alcan; 1891; Moravia, Sergio. II pensiero degli Idéologues: Scienza e filosofía in Francia (1780-1815). Firenze: La Nouva Italia; 1974; Gusforf, Georges. La conscience révolutionnaire, les Idéologues. Paris: Payot; 1978; Kennedy, Emmet. A philosophe in the Age of Revolution: Destutt de Tracy and the origins of «ldeology». Philadelphia: American Philosophical Society; 1978; Welch, Cheryl B. Liberty and Utility. The French idéologues and the transformation of liberalism. New York: Columbia University Press; 1984; Head, Brain William. Destutt de Tracy: Ideology, language, and the critique of metaphysics. In: Bloom, Harold, ed. French prose and criticism, 1790 to World War II. New York: Chelsea House Publishers; 1985, p. 7-34; Sánchez-Mejía, María Luisa. La teoría política de los ideólogos. Estudio preliminar. In: Textos políticos de los ideólogos. Madrid: Centro de Estudios Políticos y Constitucionales; 2004, p. xi-xxxviii. 
causas de las limitadas referencias o producciones sobre el tema, sino más bien esbozar algunos de sus rasgos principales en función de comprender su proyección en el espacio porteño.

La Ideología, pues, es una corriente de filosofía francesa de fines del siglo XVIII, de unos hombres que se llamaban a sí mismos idéologistes. El término deriva de la voz idéologie, neologismo creado por Destutt de Tracy para designar una «ciencia de las ideas» ${ }^{2}$. Durante la época napoleónica fue reemplazado por el de idéologues, que reflejaba una connotación despectiva. Este movimiento abarca un conjunto de pensadores muy diversos tales como Condorcet, Condillac, Siéyes, Daunnou, Volney, de Tracy, Cabanis, o La Romiguière, entre otros. Si bien existen distintos matices entre los integrantes, es cierto que todos ellos parten de un mismo fondo teórico: la idea de Condillac de reducir todo el conocimiento humano a las impresiones sensitivas $^{3}$. Así, se nuclearon en torno a una teoría sensualista del conocimiento cuya premisa básica era que las ideas, los conocimientos y todas las facultades del entendimiento humano se basan en datos sensoriales ${ }^{4}$.

Una vez desatada la Revolución, los ideólogos participaron activamente en la reconstrucción de la nueva vida política. Adhirieron al sistema republicano de gobierno, si bien se distanciaron de los jacobinos durante la época de la Convención, por sus excesos políticos, inclinándose a favor del grupo girondino. La época de mayor protagonismo del grupo se produjo con el advenimiento del Directorio (1795-1799). En las cuestiones políticas apoyaron la separación de la Iglesia y el Estado que se había decretado en febrero de 1795. Los ideólogos defendieron las iniciativas de reducir la influencia de la iglesia tanto en la política como en la educación ${ }^{5}$. Sin embargo, el panorama a su favor se perdió tras el golpe del 18 Brumario y la instalación del Consulado.

\footnotetext{
2. Para Destutt de Tracy la Idéologie se definía strictu sensu por ser la science des idées. Véase Tracy, Destutt de. Éléments d’Idéologie. Idéologie proprement dite. Première partie. Paris: Librairie Philosophique J. Vrin; 1970, p. 4-5.

3. La obra de Condillac, el Tratado de las sensaciones, publicado en 1754, impulsó los principios más generales de los Ideólogos. En ella, se sostiene que el conjunto de las facultades del hombre derivan de los sentidos, o mejor dicho, de las sensaciones. A partir de esta visión, Condillac y los ideólogos se distanciaban de la vertiente cartesiana.

4. Rejai, Mostafa. Ideology. In: Wiener, Philip Paul, ed. Dictionary of the History of ideas. Studies of Selected Pivotal Ideas. New York: Charles Scribner's Sons; 1973-74, vol. 2, p. 554.

5. Head, n. 1, p. 27.
} 
Ante la reconciliación del catolicismo como religión oficial del estado francés con la firma del Concordato de 1802, los ideólogos comenzaron a ser tratados como furiosos anticatólicos y fueron acusados de destruir la moral del hombre. En 1812 Napoleón decidió prohibir la enseñanza de la Ideología en el Instituto de Francia ${ }^{6}$. Finalmente, con una Europa asentada en los lineamientos del Congreso de Viena, los ideólogos perdieron los espacios de influencia.

En el grupo destacó la figura de Destutt de Tracy quien adquirió gran protagonismo a partir de la época consular e imperial ${ }^{7}$. Tracy logró difundir las concepciones del movimiento a partir de la publicación de su Éléments d'Idéologie, de la que aparecieron sucesivamente cuatro partes: Ideología (1801), Gramática general (1803), Lógica (1805) y Tratado sobre la voluntad (1815), seguramente su obra más difundida en Francia y América ${ }^{8}$. Su concepción estuvo centrada en desarrollar una ciencia moderna basada en una lógica racional y separada de la metafísica escolástica, a la que asociaba con el poder absolutista.

Pero fue Pierre Jean Georges Cabanis quién suministró una impronta fisiológica al movimiento incorporando al discurso filosófico un discurso médico. Cabanis nació en Cosnac (Corrèce) en 1757 y murió en 1808. Estudió medicina en París. La viuda de Helvetius hizo para él de segunda madre. A su lado le fue dado conocer a Diderot, d'Holbach, d'Alembert y Condorcet. Su método consistió en aislar los elementos, para caracterizarlos mejor y deducir diagnósticos sobre los aspectos físicos y morales del hombre. En 1802 publicó su obra mayor: Rapparts du Physique et du moral

6. Head, n. 1, p. 29.

7. Antoine Louis Claude Destutt, conde de Tracy, nació en París en 1754 y murió en 1836. Recibió una educación universitaria en Estrasburgo acorde a su rango aristocrático. Luego, ingresó al ejército realizando una carrera militar que lo llevó a obtener el grado de jefe de caballería del ejército del general Lafayette. En 1789 desempeñó el cargo de diputado en los Estados Generales representando a la nobleza, y fue un activo entusiasta de la Revolución hasta su paso a la época del Terror en 1792. A pesar de su defensa del republicanismo, fue un crítico al gobierno de Robespierre. Se salvó de la guillotina tras el golpe de Termidor en 1795 que originó con la caída de la Convención. Durante el Directorio, participó de la vida política como miembro activo del Senado. Welch, n. 1, p. 2-40.

8. Este texto se difundió ampliamente por Francia al ser el manual obligatorio en las escuelas de instrucción pública. A partir de 1820 se divulgó especialmente en Italia, Rusia e Inglaterra. También llegó a España en 1803 a través de la traducción que hizo la revista Variedades de Ciencia, Literatura y Artes. Aunque es a partir del Trienio liberal en 1821 cuando aparecieron varias traducciones de los Elementos de Ideología. Sánchez-Mejía, n.1, p. xxxi-xxxii. 
de l'Homme donde analizó las relaciones de lo moral con las edades, sexos, razas, climas. Asimismo, realizó un estudio de las sensaciones externas e internas, del sistema nervioso, de la fisiología cerebral, del pensamiento, de las pasiones ${ }^{9}$.

Mientras que la Europa de la Restauración daba la espalda a los ideólogos, la región del Río de la Plata se ofreció como un lugar abierto y fértil para experimentar sus concepciones. Un espacio que tras la declaración de la independencia y las guerras libradas a tal efecto se encontraba en pleno proceso de reorganización de la vida política y, en consecuencia, con una dirigencia que se interesó en sus principios y objetivos, para sustentar un nuevo proyecto de organización.

\section{Los antecedentes: la recepción de la Idéologie y el contexto político porteño}

La caída del sistema político del Directorio como consecuencia de la batalla de Cepeda en febrero de 1820 causó la pérdida del control de Buenos Aires sobre el resto de las provincias interiores. En los años siguientes, las Provincias Unidas del Río de la Plata carecieron de un poder político centralizado. El ascenso de los poderes provinciales se concretó con la consolidación de autonomías políticas, asentadas en el dictado de constituciones en varias de ellas, y en la aparición de caudillos que manifestaban las aspiraciones de los poderes regionales. Buenos Aires derrotada y humillada, resignó las ansias de un control social y político más englobador que había caracterizado la llamada «carrera de la revolución» de la época del Directorio, y se preparó para una organización de sus propios andamiajes institucionales en un contexto en que las condiciones de gobernabilidad eran críticas ${ }^{10}$.

La gestión de Martín Rodríguez plasmó un diseño del espacio político basado en el modelo republicano y representativo de gobierno para lo cual

9. Para profundizar remitirse a Staum, Martin S. Cabanis. Enlightenment and Medical Philosophy in the French Revolution. Princeton: Princeton University Press; 1980; Besancon, Serge. La philosophie de Cabanis, Une réforme de la psychiatrie. Paris: Institut Sinthélabo; 1997; Foucault, Michel. El nacimiento de la clínica. Buenos Aires: Siglo XXl; 2008, p. 95-123.

10. Halperin Donghi, Tulio. Revolución y guerra. Formación de una élite dirigente en la argentina criolla. Buenos Aires: Siglo XXI; 1979, p. 353. 
fue necesario introducir nuevos mecanismos institucionales ${ }^{11}$. Asimismo, se amplió la frontera política al incluir el territorio provincial. Paralelamente a la conformación de este nuevo orden político, Bernardino Rivadavia, ministro de gobierno de Martín Rodríguez, implementó un abanico de reformas institucionales en lo económico, religioso, educativo, cultural y militar. El objetivo era establecer las bases para la organización de un estado moderno afín a las concepciones ilustradas y republicanas de la época para lo cual se debía consolidar una nueva cultura política.

Si bien la crisis del orden colonial español produjo en la elite porteña un mayor interés por las obras de autores ilustrados, el mismo habría de incrementarse con la crisis del poder central, en 1820. Al respecto, Klaus Gallo indica que fue especialmente a partir «...de su encuentro con Bentham y Tracy, que Rivadavia comenzaría a trazar los principales lineamientos del modelo de gobierno y sociedad republicana que intentaría poner en marcha al regresar a la política porteña» ${ }^{12}$.

Esta apertura cultural estuvo también vinculada con los cambios en la producción y consumo porteño. A partir de 1820, la provincia experimentó una expansión económica al convertirse en proveedora de cueros para el mercado ultramarino. Este despegue económico de los primeros años de la década de 1820 contribuyó a generar un espacio más intenso en el intercambio de bienes materiales y culturales que la revolución y la guerra habían afectado. Al respecto, señala Alejandro Parada que dentro del comercio ultramarino, la importación de libros europeos contó con un incremento notable, aunque luego quedase restringido por los efectos de la guerra contra el Brasil ${ }^{13}$. Este aumento del comercio libresco fue un proceso paralelo a la necesidad de la elite de consumir productos cultura-

11. En 1821 se dictó la ley electoral y el derecho al voto «universal» que incorporaba a la campaña bonaerense. El derecho al sufragio junto a un régimen de elección directa de diputados a la Sala de Representantes — poder legislativo provincial creado en 1820 y encargado de designar al gobernador - originó una nueva concepción de la relación entre gobernantes y gobernados.

12. Gallo, Klaus. En búsqueda de la «República ilustrada». La introducción del utilitarismo y la Idéologie en el Río de la Plata a fines de la primera década revolucionaria. In: Herrero, Fabián, comp. Revolución, política e ideas en el Río de la Plata durante la década de 1810. Buenos Aires: Ediciones Cooperativas; 2004, p. 90.

13. Parada, Alejandro. El mundo del libro y de la lectura durante la época de Rivadavia. Una aproximación a través de los avisos de La Gaceta Mercantil (1823-1828). Cuadernos de Bibliotecología. 1998; 17: 12. 
les - especialmente libros y revistas- para conocer nuevas concepciones a fin de aplicarlas a la reconstrucción de la cultura política preexistente.

De la corriente ideologicista, las obras de Condillac, Condorcet, Daunnou, Destutt de Tracy y Cabanis fueron las más divulgadas en la escena local ${ }^{14}$. Un antecedente lo constituye la obra de Felipe Senillosa publicada en 1817, que menciona en su prólogo a Destutt de Tracy ${ }^{15}$. Otra referencia relevante la constituye el libro Curso Filosófico de Juan Crisóstomo Lafinur, obra difundida en sus clases de filosofía en el Colegio de la Unión del Sud hacia 1819. En este manual se puede identificar la presencia de los principios de Condillac, Destutt de Tracy y Cabanis. Sin entrar en sus detalles, es significativo apuntar que existió una polémica intensa derivada de la introducción de este nuevo tipo de enseñanza ${ }^{16}$. Es posible, pues, mencionar que esta disputa generó una primera difusión de la Idéologie en el plano local.

Este tipo de recepción hace pensar que los ideólogos fueron conocidos especialmente a través de este tipo de relecturas y de las reproducciones fragmentarias que hacía la prensa, más que por el acceso directo a sus obras. Al respecto, se registran diversas referencias en periódicos de la época tales como El Centinela (1822-1823), El Argos de Buenos Aires y Avisador Mercantil (1822-1825), La Abeja Argentina (1822-1823) y El Observador Eclesiástico (1823-1824).

\section{Los saberes fisiológicos situados en la Universidad de Buenos Aires}

A estas evidencias de la recepción del discurso ideologicista debe sumarse la producida a través de los vínculos interpersonales, los viajes y las comu-

14. Ghioldi, Delfina Varela Dominga de. Filosofía argentina. Los ideólogos. Buenos Aires: La Vanguardia; 1938, p. 62; Korn, Alejandro. Influencias filosóficas en la evolución nacional. Buenos Aires: Hachette-Solar; 1983, p. 151-166.

15. Senillosa, Felipe. Gramática española o principios de la gramática general, aplicada a la lengua castellana. Buenos Aires: Imprenta de los niños Expósitos; 1817.

16. Estas polémicas se encuentran en Gutiérrez, Juan María. Noticias históricas sobre el origen y el desarrollo de la enseñanza superior en Buenos Aires 1868. Buenos Aires: Universidad Nacional de Quilmes; 1998, p. 122-123. Para profundizar este asunto, véase Gallo, Klaus. A la altura de las luces del siglo: el surgimiento de una clima intelectual en la Buenos Aires posrevolucionaria. In: Altamirano, Carlos, dir. Historia de los intelectuales en América Latina. Tomo I: La ciudad letrada, de la conquista al modernismo. Madrid: Katz editores; 2008, p. 184-204. 
nicaciones informales que también reflejan el intercambio y la circulación de saberes. Al respecto, resulta sugerente considerar la correspondencia entre Destutt de Tracy y Bernardino Rivadavia ${ }^{17}$. El intercambio epistolar refleja un lenguaje de naturaleza coloquial que remite a un conocimiento previo y personal producto de la estadía de Rivadavia en Francia en años anteriores ${ }^{18}$. En esa oportunidad, se conformaron una serie de contactos y redes sociales que explican la existencia de una comunicación fluida de ideas, noticias y saberes, reforzada hacia 1824 cuando Rivadavia regresó a Europa.

El análisis de la correspondencia pone de manifiesto que las nociones de Destutt de Tracy atrajeron a la dirigencia porteña para resolver un tema central: modernizar la educación en todos sus niveles. El 19 de junio de 1821 Rivadavia fue nombrado Prefecto del departamento de estudios preparatorios, cargo desde el que preparó el camino para la institucionalización de la cátedra. Sin embargo, recién cuando dejó ese cargo para ocupar el Ministerio de gobierno, durante la gestión de Martín Rodríguez, el 19 de julio de ese mismo año, pudo concretar su intención. El 9 de agosto firmó junto al gobernador el Edicto de creación de la Universidad de Buenos Aires. La estructura departamental del nuevo organismo se realizó agrupando distintas instituciones heredadas de la época colonial aunque se introdujeron modificaciones, entre ellas la inclusión de la cátedra de Ideología ${ }^{19}$. Esta se incorporó como materia obligatoria del plan de estudios preparatorios dictándose en el Colegio de Ciencias Morales que dependía de la Universidad de Buenos Aires ${ }^{20}$.

En una de las cartas, Destutt de Tracy escribe a Rivadavia:

«A la verdad, es una bella institución la de una cátedra de Economía política y otra de Ideología. En cuanto a esta última yo me considero feliz, si mi obra puede ser de alguna utilidad, entre tanto no aparezca otra mejor» ${ }^{21}$.

17. La mayoría de las cartas se encuentran editadas en Picirrilli, Ricardo. Rivadavia y su tiempo. 2 Vols, Buenos Aires: Ediciones Peuser; 1943.

18. Sobre la gestión de Rivadavia en Europa entre 1814 y 1820, véase Gallo, n. 12, p. 89.

19. La Universidad de Buenos Aires quedó organizada en seis departamentos: el de primeras letras, el de estudios preparatorios, el de ciencias exactas, el de medicina, el de jurisprudencia y el de ciencias sagradas. Véase Gutiérrez, n. 16, p. 274.

20. El Colegio de la Unión del Sur se transformó en el Colegio de Ciencias Morales hacia 1823, anexándose a la Universidad.

21. Carta de Destutt de Tracy a Bernardino Rivadavia. París, 18 de noviembre de 1822. Museo Mitre, Colección Correspondencia Hombres Públicos Argentinos, ref. 1C22C16, Nro. 1, folio 15925. 
Según el pensador francés, la intención de Rivadavia residió en «acrecentar y esparcir las luces entre los ciudadanos» ${ }^{22}$. Una vez dominado el clero, la institución se convirtió en un agente libre para difundir los nuevos saberes. En el caso de los estudios preparatorios, los saberes sensualistas debían fijar un nuevo rumbo desplazando la enseñanza de la filosofía escolástica.

Durante la gobernación de Martín Rodríguez (1821-1824), seguida por la de Las Heras (1824-1825), la presidencia de Rivadavia (1826-1827) y la gobernación de Dorrego (1827-1828) circuló el texto Principios de Ideología que confeccionó Juan Manuel Fernández de Agüero, primer profesor de la cátedra. La aparición de este escrito pedagógico es un indicio para sostener que la difusión del discurso sensualista fue adquiriendo mayor presencia en aquellos momentos. En los Principios de Ideología existen reiteradas menciones a Destutt de Tracy. Las continuas referencias explícitas permiten inferir que el profesor examinó directamente los Éléments d'idéologie ${ }^{23}$.

La situación incierta sobre la conclusión de la Guerra con el Brasil, la presión de los nuevos grupos rurales para la obtención de más cargos públicos, la oposición de sectores reacios a los cambios introducidos, como el clero y el ejército, la reacción de las provincias ante la Constitución unitaria de 1826, entre otras cuestiones, motivaron la caída de la administración rivadaviana hacia 1827. En Buenos Aires, Manuel Dorrego fue elegido gobernador y con él se introdujeron los principios del federalismo en la escena provincial. No obstante, fue destituido rápidamente por la invasión llevada a cabo por el general unitario, Juan Lavalle. Con la muerte de Dorrego, en manos de Lavalle, se generó una crisis política que posibilitó el ascenso de Juan Manuel de Rosas a la gobernación de la provincia ${ }^{24}$.

De las características principales del sistema político rosista podríamos destacar la continuidad del ejecutivo y la centralización del poder. Bajo el

22. Carta de Destutt de Tracy a Bernardino Rivadavia. París, 20 de septiembre de 1823. Reproducida en Piccirilli, n. 17, vol. 1, p. 488.

23. Fernández de Agüero sostiene con respecto a los Éléments d'idéologie: «Ya en la primera lectura rápida que habíamos hecho de esta obra le habíamos notado...». Véase Fernández de Agüero, Juan Manuel. Principios de Ideología Elemental, Abstractiva y Oratoria. Tomo I. Buenos Aires: Universidad de Buenos Aires; 1940, p. 137.

24. Pagani, Rosana; Souto, Nora y Fabio Wasserman. El ascenso de Rosas al poder y el surgimiento de la Confederación (1827-1835). In: Goldman, Noemí, dir. Revolución, república, confederación (1806-1852), tomo III, Nueva historia argentina. Buenos Aires: Sudamericana; 1998, p. 283-380. 
régimen rosista la sociedad se polarizó por el nivel de adhesión o rechazo político con respecto de la gestión de gobierno. Pero cabe indicar que no se desarticuló el andamiaje republicano y representativo introducido por las reformas rivadavianas, sino que éste fue reordenado bajo otra modalidad ${ }^{25}$. Asimismo, durante la primera y segunda gobernación de Rosas se produjo una participación activa de los sectores populares y una alta politización del conjunto social ${ }^{26}$. La beligerancia permanente contra todos los intentos de oposición, sumado a las guerras entabladas con potencias extranjeras, generaron una situación de constante inestabilidad política ${ }^{27}$.

En este entramado particular se insertó la vida intelectual y académica. El proceso de politización de la sociedad, sumado a las guerras internas y externas a lo largo del período, tuvo un impacto significativo en la universidad porteña, en cuyos recintos se experimentaron una serie de divisiones, conflictos y opiniones encontradas. Cabe indicar que la universidad siguió funcionando pero de forma muy limitada dada la escasez de estudiantes, las presiones facciosas, las renuncias y despidos de profesores, y la falta de financiamiento por parte del gobierno ${ }^{28}$.

Sin embargo, los saberes ideologicistas fueron paulatinamente emergiendo en los ámbitos académicos y en la opinión pública. Proceso que adquirió una mayor intensidad y un giro hacia la vertiente fisiológica a través del impulso dado por el flamante profesor de Ideología, luego del alejamiento de Fernández de Agüero en 1827, el Dr. Diego Alcorta.

\section{Diego Alcorta y la apropiación del lenguaje fisiológico}

Diego Alcorta nació en Buenos Aires, el 11 de noviembre de 1801, siendo hijo de don Juan Bautista Alcorta y de doña María Elena Ramírez. Cursó sus estudios en el Colegio de la Unión de Sur donde asistió a las lecciones de

25. Nos referimos al paso del régimen de competencia entre notables al plebiscitario. Véase Ternavasio, Marcela. Las reformas rivadavianas en Buenos Aires y el Congreso general constituyente. In: Goldman, n. 24, tomo III, p. 161-197.

26. Di Meglio, Gabriel. jViva el bajo pueblo! La plebe urbana de Buenos Aires y la política entre la revolución de mayo y el rosismo. Buenos Aires: Prometeo; 2006, p. 225-307.

27. Myers, Jorge. Orden y virtud. El discurso republicano en el régimen rosista. Buenos Aires: Universidad Nacional de Quilmes; 1995, p. 20.

28. Buchbinder, Pablo. Historia de las universidades argentinas. Buenos Aires: Sudamericana; 2005, p. $48-49$. 
filosofía a cargo del profesor Crisóstomo Lafinur, quien le habría despertado gran interés por esta disciplina ${ }^{29}$. En 1824, durante su época de estudiante, fundó, junto a un grupo de compañeros, la Sociedad Elemental de Medicina, en donde cada uno de los socios obtenía un número de orden equivalente a su sitial académico. Entre ellos se encontraba Juan José Montes de Oca, primer promedio de su promoción, y futuro profesor titular de clínica quirúrgica y decano de la Facultad de Medicina. Se realizaban sesiones semanales en la que cada uno de sus miembros presentaba diferentes temas. De esta forma, Alcorta expuso sobre afecciones patológicas combinadas de los órganos y sobre las variedades del pulso arterial ${ }^{30}$.

Posteriormente fue nombrado practicante mayor rentado del Hospital General de Hombres, lo cual le permitió mejorar su precaria situación económica. En 1827 finalizó sus estudios recibiendo el título de doctor en medicina con un trabajo de tesis titulado Disertación sobre la Manía Aguda. En 1828, fue nombrado médico del Hospital de Hombres especializándose en cirugía y propuesto profesor titular de la cátedra de Ideología en la Universidad de Buenos.

Tuvo un paso fugaz por la política. Fue electo para la Legislatura de Buenos Aires por el partido de San Isidro en 1832. Se opuso a la re-elección de Rosas en 1834, sin que esta decisión le ocasionara complicaciones. No obstante, Alcorta decidió alejarse de la política partidaria y dedicarse solamente a la enseñanza y al ejercicio de la medicina permitiéndole sostenerse en la cátedra hasta 1842 .

A partir del análisis de su tesis es posible rastrear ciertas conexiones acerca de los saberes provenientes de la Idéologie. En la Disertación, estudio basado sobre seis pacientes internados en el Hospital General de Hombres, emerge un discurso sensualista y fisiológico respecto del entendimiento de las facultades humanas. Mientras que Crisóstomo Lafinur y Fernández de Agüero retomaban el esquema de las sensaciones de Destutt de Tracy, Diego Alcorta se inclinaba por el propuesto por Pierre Jean George Cabanis en su Rappors du phisique et du moral de l'homme ${ }^{31}$. Cabe señalar que en la época por el concepto fisiología se entendía cierta retórica en torno el

\footnotetext{
29. Gutiérrez, n. 16, p. 104.

30. Alcorta, Diego. Diario literario por la Sociedad Elemental de Medicina, 1821.

31. Cabanis, Pierre Jean Georges. Rapports du physique et du moral de I'homme. Paris: L'Harmattan; 2005, vol 1, p. 203-214.
} 
entendimiento del aspecto físico y moral del hombre, aún no constituía una especialidad de la medicina.

Siguiendo de cerca al fisiólogo francés, Alcorta sostenía que lo primero en estudiarse debía ser el aspecto físico del hombre: identificar las partes del cuerpo y su organización, como así también determinar el funcionamiento de los distintos órganos porque son los que generan nuestros sentidos. El argumento puede sintetizarse de la siguiente manera: si no se consideran los sentidos, el hombre no tendría acceso al entendimiento y a captar la realidad. Este principio parte de la idea de que si estuviéramos desprovistos de manos, ojos, oídos, etc., nada podríamos conocer. Así, pues, sostenía que las enfermedades mentales eran el producto de las alteraciones orgánicas ${ }^{32}$.

Sin embargo, luego expresaba que «se ha descubierto por un gran número de disecciones comparadas que a sus enfermedades corresponden frecuentemente alteraciones en las facultades morales» ${ }^{33}$. En esta línea, también comentaba:

«Las distintas épocas de la vida vienen acompañadas del desarrollo particular de algunas de las facultades intelectuales, y de ciertos sentimientos interiores nacidos del estado actual de los órganos de la economía» ${ }^{34}$.

Es significativo observar cómo estas consideraciones aparentemente contradictorias, en realidad pretendían articular el funcionamiento biológico y orgánico de los sujetos respecto de los comportamientos sociales y morales, y viceversa. Las enfermedades mentales, como el caso de la manía, atravesaban el aspecto físico y moral en un mismo nivel.

No obstante, es cierto que existían dos niveles superpuestos de aproximación: el registro orgánico permitía diagnosticar y estudiar la enfermedad en el paciente y, el registro moral, era decisivo para comprender las causas de la misma ${ }^{35}$. Bien lo expresa Alcorta cuando indica que «toda impresión demasiado fuerte, tanto física como moral, puede determinar la manía; pero para ello es necesaria una predisposición individual que o es originaria $u$ ocasionada por la educación, la edad, la manera de vivir, el sexo, etc.» ${ }^{36}$.

32. Alcorta, Diego. Disertación sobre la manía aguda. Universidad de Buenos Aires; 1827. Localizada en: Biblioteca Nacional, Sala Tesoro, ref. 3A232210.

33. Alcorta, n. 32.

34. Alcorta, n. 32.

35. Cabanis, n. 31, p. 161-184.

36. Alcorta, n. 32. 
En este punto, Alcorta introduce algunas aseveraciones de Philippe Pinel (1755-1826), célebre médico francés cuyos saberes originaron la legislación psiquiátrica francesa de 1838. Por un lado, Alcorta retomaba de Pinel la premisa de buscar en los tejidos la localización de las enfermedades ${ }^{37}$, principio también compartido por el vitalismo o la denominada Escuela de Montpellier ${ }^{38}$. Por ejemplo, para explicar los primeros síntomas en la manía aguda Alcorta observaba que:

«Se hace también conocer este período por ciertas mudanzas singular en el color y rasgos de la fisonomía, generalmente por una debilidad extrema de los órganos de los sentidos, principalmente de la vista y del oído; por una sucesión rápida y una gran inestabilidad de ideas, a veces todas las facultades del entendimiento están trastornadas, a veces se presenta una o dos solamente» ${ }^{39}$.

Por el otro, Alcorta incorporó la clasificación pineliana de las enfermedades mentales expresada en el Traité médico-philosophique sur l'aliénation mentale ${ }^{40}$. En este libro, el médico francés distinguía entre la melancolía simple (delirio parcial), la manía (delirio generalizado con agitación), la demencia (debilitamiento intelectual generalizado) y la idiocia (perturbación total de las funciones intelectuales). Éste consideraba a las enfermedades mentales

37. Pinel, Philippe. Nosographie philosophique ou la méthode d'analyse apliqué a la médicine. París: Crapelet; 1798, vol. 2, p.160-162.

38. El vitalismo se caracterizaba por postular la existencia de una fuerza o impulso vital sin el que la vida no podría ser argumentada. El principal difusor del vitalismo en Francia fue Xavier Bichat. Este entiende que los fenómenos biológicos tienen por causa propiedades vitales innatas a todos los seres vivos. De allí deriva el límite que impondrá en sus estudios: observará los hechos sin profundizar más sus investigaciones, pues está convencido de que ni la física ni la química podrán interpretar la vida. Esta postura lo llevará hasta desconfiar del uso del microscopio, considerando la disección como forma de demostración e impulsando los estudios anatómicos. Partiendo de estas bases llega a la conclusión de que el elemento común de los órganos animales son los tejidos, elementos simples, y por lo tanto, irreductibles, los que se propone identificar. Lecourt, Dominique. Dictionnaire de la pensée médicale. Paris: Press Universitaires de France; 2004, p. 1208-1211.

39. Alcorta, n. 32.

40. Esta obra de Pinel pareciera que tuvo un peso importante en el desarrollo del pensamiento y la formación de Diego Alcorta. Véanse Conti, Norberto. Juan Manuel Fernández de Agüero y Diego Alcorta: Ideología y Locura en el Río de la Plata. Temas de Historia de la Psiquiatría Argentina. Buenos Aires: Polemos; 1997, vol. 2, p. 3-47; Stagnaro, Juan Carlos. Diego Alcorta y la manía aguda: preliminares de la psiquiatría argentina. Revista argentina de psiquiatría Vértex. 1990; 1 (1): 57-63; Balbo, Eduardo. Introduction. Dissertation on acute mania by Diego Alcorta. History of psychiatry. 1991; 2: 181-217; Mahieu, Eduardo. Diego Alcorta: Dissertation sur la manie... aiguë? Electroneurobiología. 2005; 13 (3): 283-298. 
como un desarreglo de las facultades cerebrales causado por cierto número de causas: estas podían ser físicas (directamente cerebrales o simpáticas); heredadas; y morales, a las que le atribuye más de la mitad de los casos, que se pueden separar en las que derivan de las pasiones intensas y las que provienen de los excesos de todo tipo ${ }^{41}$. Al respecto, Alcorta adaptaba esta clasificación en los siguientes términos:

«Es necesario aislar los objetos para poder conocerlos bien. He ahí la necesidad de una clasificación en las enfermedades mentales; y no pudiendo hacerla por las alteraciones orgánicas que las ocasionan, por no ser bien conocidas, es preciso hacerla por los síntomas que las caracterizan; a mi juicio, la de Pinel merece la preferencia. El distingue la enajenación mental en cuatro especies distintas: manía, melancolía, demencia e idiotismo; cada una de estas especies es susceptible de infinitas variedades. La manía, la más común de las enfermedades mentales, reclama por esta razón una atención particular» ${ }^{42}$.

La taxonomía propuesta, fruto de reducir y aislar las partes del cuerpo, se construye a partir de la posibilidad que tiene el médico de observar los síntomas. Pero, ¿qué es lo que finalmente éste puede observar para fundamentar su diagnóstico? Como parte de su labor pedagógica, Alcorta confeccionó una guía para el dictado de las clases denominada Lecciones de filosofía, obra posteriormente editada en $1835^{43}$. Dividió el manual en tres partes: Lógica, Metafísica y Retórica. En la primera parte de la Lógica, denominada «Idea general del hombre físico», detectamos la posible respuesta cuando explicaba que:

«Nuestro cuerpo está encerrado en un envoltorio general que es la piel, o el cutis, bajo la cual se hallan las carnes o músculos. Bajo de esta está la estructura ósea o sólida que lo sostiene. Esta presenta tres cavidades; que son la cabeza, el pecho o tórax, el vientre o abdomen. En la cabeza está encerrada la masa cerebral de la que una parte se llama cerebro y la otra cerebelo: una y otra reuniéndose, forman una especie de cola llamada médula oblongada. De la médula oblongada nace la médula espinal que desciende por el interior

41. Pinel, Philippe. Traité Médico-Philosophique sur l'aliénation mentale, ou la manie. Paris: Chez J. Ant. Brosson; 1809, p. 135- 137.

42. Alcorta, n. 32.

43. Alcorta, Diego. Lecciones de filosofía. Buenos Aires: Fondo Nacional de las Artes; 2001. Esta obra de Diego Alcorta se origina de apuntes manuscritos que utilizaba al dictar sus clases. Posteriormente, se editarán por primera vez recién en 1902. Groussac, Paul. Curso de Filosofía de Diego Alcorta. Anales de la Biblioteca. Tomo II. Buenos Aires; 1902. 
del canal vertebral o espinazo y termina por expansiones. De la masa cerebral y de la médula espinal parten cuarenta partes de nervios que van siempre decreciendo, que se ramifican en todo el cuerpo y terminan en los órganos y principalmente en la piel y los músculos» ${ }^{44}$.

De tal manera, los actos y comportamientos morales dependen de la formación de ideas generadas por el funcionamiento de los órganos internos, en especial, por el cerebro que comanda las impresiones sensibles a través del sistema nervioso. La enfermedad aparece por el mal funcionamiento de los órganos. El médico puede proporcionar un diagnóstico y un tratamiento, no sólo a partir de cotejar los comportamientos morales del paciente sino también observando las manifestaciones orgánicas que se revelan en los tejidos en tanto órganos superficiales del cuerpo.

En suma, Alcorta pone en relieve, a partir de su tendencia hacia las obras de Cabanis y Pinel, un lenguaje fisiológico más evidente que sus predecesores en cuestiones de filosofía. Ahora bien, ¿cómo afectaron estas nociones su práctica médica? ¿Estas ideas decidieron una perspectiva clínica? ¿Qué impacto tuvo en la enseñanza y ejercicio de la medicina local?

\section{Diego Alcorta y la construcción de una medicina sensualista, práctica y científica}

Si consideramos los años anteriores, desde la apertura del Protomedicato hasta la creación del Instituto Médico Militar —entre los años de 1776 hasta 1815-, se puede inferir que la medicina en Buenos Aires era concebida como un campo de conocimientos amplios: un médico debía recibir estudios de física, lógica, agricultura, botánica, química vegetal, curtiembre, mineralogía, meteorología, entre otras áreas ${ }^{45}$. El énfasis en la utilidad de los saberes de otras disciplinas distintas de la específica se manifestaba en

44. Alcorta, n. 43, p. 34-35. Véase también Cabanis, n. 31, p. 203-233. Hoy sabemos que tanto Cabanis como Alcorta estaban errados: el hombre tiene doce pares de nervios craneanos y treinta y un pares de nervios medulares.

45. Recordemos que, en este período, los estudios médicos duraban seis años, distribuidos de la siguiente manera: en el primero, se enseñaba anatomía; en el segundo, química farmacéutica y botánica; en el tercero, materia médica; en el cuarto, enfermedades; en el quinto, operaciones; y el sexto consistía en lecciones de medicina clínica. 
el hecho de que las oportunidades educativas eran escasas y se esperaba una formación plástica y adaptable a múltiples fines ${ }^{46}$.

Esta formación integral en la preparación de los galenos porteños comenzó a modificarse hacia 1821. Con la creación del departamento de Medicina en el marco de la Universidad de Buenos Aires, las ideas sensualistas que introdujo Diego Alcorta constituyeron un factor relevante en el proceso de construcción de un perfil más específico, científico y profesional de la medicina porteña ${ }^{47}$. En este sentido, la introducción de autores franceses representa una variación respecto de los textos académicos y manuales utilizados usualmente en la enseñanza de la medicina en el período anterior ${ }^{48}$.

En este marco de movimiento dinámico de saberes, la medicina porteña se esforzó, siguiendo el modelo europeo, en adaptarse al conocimiento científico, tal como señaló Alcorta: «En el tratamiento de ninguna enfermedad tiene el médico tanta necesidad de las luces de la filosofía como en el de la manía» ${ }^{49}$. El saber médico necesitaba de la filosofía moderna para adquirir un verdadero status científico, por esto el profesor comenta que:

«Los médicos modernos, libres de las trabas [...] miran a la inteligencia como la función de un órgano; ayudados de las luces de la anatomía y patología, ellos procuran saber su mecanismo; se hacen ensayos por todas partes, y quizá no está lejos la época en que nuevas luces adquiridas a este respecto

46. Asúa, Miguel de. La ciencia de Mayo. La cultura científica en el Río de la Plata, 1800-1820. Buenos Aires: Fondo de Cultura Económica; 2010, p. 159.

47. Para la cuestión de la profesionalización del campo médico en Europa y los Estados Unidos, entre otros, véanse Ramsey, Matthew. Professional and Popular Medicine in France 1770-1830: The Social World of medical practice. Cambridge: Cambridge University Press; 1988; Haber, Samuel. The quest for authority and honor in the American professions, 1750-1900. Chicago and London: University of Chicago Press; 1991; y Bonner, Thomas. Becoming a physician: medical education in Britain, France, Germany, and the United States 1750-1945. Oxford: Oxford University Press, 1995. Para el caso argentino, remitirse a González Leandri, Ricardo. Curar, persuadir, gobernar. La construcción histórica de la profesión médica en Buenos Aires (1852-1886). Madrid: CSIC; 1999; Di Liscia, María Silvia. Saberes, terapias y prácticas médicas en Argentina (1750-1910), Madrid: CSIC; 2002.

48. El plan de estudios se basaba en el de la Universidad de Edimburgo. Para la enseñanza de medicina se usaban los textos de William Cullen y James Gregory. Para cirugía se recomendaban textos españoles, como el Curso completo de anatomía de Jaime Bonells e Ignacio Lacaba, de cinco tomos, y las Operaciones de cirugía de Francisco Villaverde. Cantón, Eliseo. La facultad de Medicina y sus escuelas. Tomo I, La medicina, su ejercicio y enseñanza en el pasado colonial y en la Independencia (1580-1821). Buenos Aires: Coni; 1921, p. 235-240.

49. Alcorta, n. 32. 
hagan tomar a la medicina un grado de certidumbre en las enfermedades mentales de que hasta ahora carece notablemente ${ }^{50}$.

«Libres de las trabas» representaba la posibilidad de ensayar ciertos procedimientos específicos para mejorar los estudios anatómicos como por ejemplo examinar los cadáveres humanos sin reprobación alguna. De allí la necesidad de construir un conocimiento basado en certezas comprobables. Para ello, por un lado, habría que apartarse de las explicaciones religiosas y teológicas preponderantes en la etapa colonial y de aquellos conocimientos «dudosos» $\mathrm{y}$ «populares» derivados de las culturas originarias y los sectores bajos; por el otro, diferenciarse de las demás disciplinas convergentes tales como la química, la botánica, la física y la farmacéutica.

En cuanto al impacto profesional en el mismo ejercicio médico, Alcorta retomó de la escuela sensualista, en especial, la importancia de la observación como método de diagnóstico y curación en el tratamiento de las enfermedades y, la inauguración, en la enseñanza e investigación médicas, de una orientación empírica que introdujo una actividad práctica específica: la clínica hospitalaria. Al respecto, existen varios casos prácticos relevantes. Uno de ellos corresponde al propio Alcorta en el Hospital Mayor de Hombres que nos sirve para constatar cómo estos métodos empezaban a aplicarse en la escena local:

«Jacinto Roque Arias natural de Córdoba de edad de 23 años y de un temperamento sanguíneo hacia 10 años que comenzó su enfermedad; su origen fue una herida hecha con instrumento cortante y punzante, situada en la parte inferior interna y un poco posterior en el muslo derecho: ella se dirige oblicuamente hacia delante y hacia adentro. Este individuo estuvo por tres veces a punto de perder la vida a consecuencia de hemorragias repetidas y difíciles de contener. Se observó un pequeña tumor en la parte interna en el muslo que desapareció por la compresión: finalmente el salvó y no sintió novedad hasta los dos años en que se dejaron sentir dolores insoportables, acompañados en el hinchazón y hematomas en la pierna: empezaron a hincharse las venas de ella, y reventándose algunas dieron origen a dos úlceras varicosas. Se observó en la parte interna e inferior del muslo una pudrición particular que sucesivamente se fue extendiendo hacia la ingle y la cava. Hace 15 días que sobrevino un tumor doloroso un poco más abajo en la ingle. He ahí la historia de la enfermedad. En la actualidad el enfermo se encuentra con 
los síntomas siguientes: hinchazón edematosa en la pierna y muslo derecho, las venas se encuentran varicosas, dos ulceras en el mismo carácter en la parte inferior de la tibia. En todo el trayecto de la arteria femoral se presenta una pudrición singular más fácil de sentir que de explicar: ella se puede comparar a la sensación que presentarían muchos pequeños canales a la vez, acompañada de un fluido semejante al que produce un líquido empujado en un canal estrecho, y que encuentra una abertura angosta» ${ }^{51}$.

Alcorta busca precisar, anotar, registrar, día a día, los cambios de la enfermedad en el cuerpo del paciente y diagnosticar las causas de la enfermedad a través de los síntomas observados en los músculos, las venas y los tejidos: es decir, las ramificaciones más externas de los órganos internos. El pronóstico es mortal, por ello Alcorta contraindica la operación de ligar la arteria dañada e incluso también desestima la amputación. En el tratamiento lo que le queda al médico es suavizar los días amargos que le restan al paciente.

Pero cabe preguntarse: ¿qué ocurrió con estos procedimientos prácticos y la difusión de los nuevos saberes fisiológicos más allá de Diego Alcorta? Para responder a esta pregunta compleja se ha realizado un relevamiento de las tesis presentadas y defendidas en la Facultad de Medicina en la Universidad de Buenos entre 1827 y 1850, lo cual nos permite inferir ciertas huellas dejadas por el lenguaje ideologicista y fisiológico e intentar medir su impacto dentro del mismo ejercicio de la medicina.

Los períodos de crisis económicas y políticas coinciden con la baja producción de tesis. Por otro lado, es interesante indicar que existen dedicatorias referidas a asuntos públicos producto de la alta politización que mencionábamos más arriba como lo sugieren las siguientes inscripciones: « ¿Viva la Santa Confederación Argentina!», «iMueran los salvajes Unitarios!», «iMuera el loco traidor salvaje unitario Urquiza!», entre otros lemas. Aquí repararemos en algunos casos significativos, cuyo rasgo común es que son estudios apoyados y derivados de la observación y la práctica clínica ${ }^{52}$.

51. Alcorta, Diego. Hospital General de Hombres, Sala Primera, Cama Número 33, 1827. Localizado en: Biblioteca Nacional, Sala del Tesoro, Colección Candiotti, Tomo I, 1827-1832, ref. 3A63519.

52. En este asunto, agradezco la colaboración de Karina Ramacciotti. En pos de brindar una dimensión más amplia del nivel de difusión deberíamos incorporar otras tesis defendidas en la primera mitad del siglo XIX, pero por cuestiones de espacio sólo indicamos que se encuentran alojadas en la Biblioteca de la Facultad de Medicina de la Universidad de Buenos Aires y en la Sala del Tesoro de la Biblioteca Nacional. En el primer reservorio se registran unas 246 tesis, pero solo se puede precisar autor y tema de 58. De esta cantidad, la Biblioteca sólo 
Para obtener su grado de doctor en medicina, Juan Gutiérrez Moreno escribió su Thesis sobre la rabia en 1830 donde manifestaba:

«La invasión, síntomas, y demás fenómenos enumerados inclinan el ánimo a favor de la opinión, que establece el asiento de la rabia en el sistema nervioso; el aumento de sensibilidad de relación expresa la exagerada sensibilidad de los nervios de los sentidos, y las alucinaciones que experimentan, una aberración de las propiedades sensitivas, ya de los nervios que la impresión trasmiten, o bien del centro, o punto de convergencia donde terminan todas las sensaciones ${ }^{53}$.

El razonamiento ideologicista y fisiológico emerge de la apelación constante a la cuestión de la sensibilidad. Es pertinente resaltar cómo en estas argumentaciones sobre los efectos de la rabia cobra importancia considerar a los sentidos y sus derivaciones: esta enfermedad incrementa el funcionamiento del aparato sensorial. La rabia a su vez ocasiona alucinaciones que alteran la actividad de los nervios, y sobre todo, al órgano principal, el cerebro. Asimismo, pueden verse semejantes fundamentaciones en la tesis de Claudio Mamerto Cuenca, Las simpatías en general, consideradas en sus relaciones con la anatomía, fisiología, patología y terapéutica, defendida en 1838, cuando indicaba que:

«En efecto, las enfermedades no corren tan rápida, ni pasan tan superficialmente sobre los órganos, que no dejen rastros más o menos aparentes de su existencia. Saber, pues, apreciar esos desordenes orgánicos que acompañan, o mejor, que constituyen las enfermedades, importa saber apreciar la causa próxima que las entretiene, cuyo conocimiento es el secreto maravilloso del arte de curar» ${ }^{54}$.

Asimismo, el lenguaje fisiológico difundido por los ideólogos locales se registra en la tesis de José Lucena. En 1848, Lucena obtenía su grado de

posee 36 tesis completas, 16 manuscritas y 16 impresas o mecanografiadas. En el caso de la Biblioteca Nacional, las tesis se encuentran bajo la Colección Candiotti, pero las referidas a medicina corresponden sólo al período comprendido entre 1827 y 1832.

53. Gutiérrez Moreno, Juan. Thesis sobre la rabia. Universidad de Buenos Aires; 1830. Localizada en: Biblioteca Nacional, Sala del Tesoro, ref. 3A232210.

54. Cuenca, Claudio Mamerto. Opúsculo sobre las simpatías en general, consideradas en sus relaciones con la anatomía, fisiología, patología y terapéutica. Universidad de Buenos Aires; 1838. Tesis doctoral localizada en: Biblioteca de la Facultad de Medicina, Universidad de Buenos Aires, ref.: 22522. 
doctor en medicina con un estudio titulado Sobre el histerismo, considerada como una enfermedad nerviosa. Su argumento principal era demostrar que la histeria estaba unida a determinados tipos de comportamientos y prácticas morales:

«El temperamento nervioso y una constitución pletórica, la dismenorrea, la amenorrea son causas predisponentes al histerismo, a estas es preciso unir un amor contrariado, los celos, la influencia de las lecturas obscenas, las consideraciones propias para ideas lascivas, lo mismo que la continencia, mientras otras ocasiones los abusos venéreos. Es una impresión moral viva, la supresión de las reglas se considera como causas excitantes de la enfermedad [... $»^{55}$.

Si bien la histeria no era concebida como una enfermedad mental, como en el caso de la manía aguda que aparece en la Disertación de Alcorta, las alteraciones orgánicas se corresponden con las conductas morales y, por ende, éstas últimas podrían constituir el origen de la enfermedad. Aquí Lucena repara en ordenar y agrupar los síntomas hasta encontrar cuadros uniformes. En cuanto a la terapéutica, siguiendo de cerca a Pinel, proponía el manipuleo de las pasiones del paciente, hasta calmarlo por medio de un «tratamiento moral». Este «tratamiento moral» consistía en aplicar al enfermo una serie de ingenuos ardides como trabajos agrícolas y/o escuchar música para demostrar lo errado de sus juicios, a las que se agregaban otro conjunto de medidas como sangrías para la retención de sangre menstrual o hemorroidal, purgantes suaves o baños templados.

\section{Conclusiones}

Este trabajo realiza una primera aproximación a la difusión de los saberes médicos en Buenos Aires, en especial los producidos por la introducción de la Idéologie en el ámbito universitario. Se ha registrado un intenso intercambio de saberes que pone de manifiesto el proceso de apropiación porteño de un discurso filosófico y médico europeo. Esta labor de apropiación intelectual comprende la reelaboración de percepciones e ideales, así como el establecimiento de normas de diversa índole. Tal proceso se revela

55. Lucena, José. Sobre el histerismo, considerada como una enfermedad nerviosa. Universidad de Buenos Aires; 1848. Tesis doctoral localizada en: Biblioteca de la Facultad de Medicina, Universidad de Buenos Aires, ref.: 23244. 
también, de forma hipotética, como creador de una lógica de diferenciación, portadora, a veces, de una conciencia de la alteridad de un mundo percibido hasta entonces sólo como el aprendiz de la metrópoli.

En este marco, se ha analizado en especial a Diego Alcorta. Entre sus intenciones iniciales, se encontraba el reemplazo de la filosof ía escolástica aún vigente en la época. Pero su contribución más significativa se produjo en el ámbito médico al incorporar un lenguaje sensualista y fisiológico y una práctica médica asentada en los estudios clínicos. En este sentido, los primeros pasos dados por Crisóstomo Lafinur y Juan Manuel Fernández de Agüero en la cátedra continuaron a través de las enseñanzas de Diego Alcorta a partir de 1828, quien otorgó un rasgo fisiológico más evidente al incorporar las nociones de Philippe Pinel y las provenientes de Jean Pierre Georges Cabanis.

Así, pues, Alcorta logró presentar un discurso que intentaba la articulación de lo orgánico y lo humano, de lo físico y lo moral, en definitiva, de la medicina y la filosofía. En estas teorías subsiste un eco de lo que Marcel Gauchet analiza en su obra Le désenchantement $d u$ monde: la idea que el origen de la vida se encuentra en la materia misma y no en una fuerza exterior a ella ${ }^{56}$.

Los médicos aportaron su concurso y su caución a esta dinámica de apropiación de ideas referidas a la salud y la enfermedad, al tiempo que encontraron allí un importante vector de promoción. No es sorprendente que la incipiente medicina porteña pusiera su vista en estos saberes que le ofrecían, por un lado, una teoría fisiológica que conectaba las funciones de la mente con el cuerpo, y, por otro, la posibilidad de legitimarse como ciencia moderna sin la intervención eclesiástica en estas materias. En tal sentido, Alcorta pretendió desarrollar, en la enseñanza y en la práctica profesional, métodos concretos e empíricos: la clínica hospitalaria y la observación anatómica.

A partir de la exploración de este problema se podría constatar también la creciente importancia de la figura del médico en el espacio porteño. Hacia la década de 1830 la medicina comenzaba a considerarse una actividad científica, académica y profesional. Asimismo, estos saberes contribuyeron a delinear una mirada de lo político imbuida en un tipo de lenguaje fisiológico

56. Gauchet, Marcel. Lé désenchantement du monde. Une histoire politique de la religion. Paris: Gallimard; 1985. 
y biológico. La ética y la política serían ideología y fisiología aplicada. Ambas no hacían más que demostrar la derivación de los sentimientos morales y sociales (odio o simpatía) de las impresiones sensibles y, por consiguiente, de las condiciones del sistema nervioso.

\section{Agradecimientos}

Agradezco las orientaciones y sugerencias de Karina Ramacciotti y Ricardo González Leandri, como así también a mis directores de tesis doctoral, Pilar González Bernaldo y Jaime Peire. Por último, mi reconocimiento a Diego Fracchia y a Lourdes Braña, por la colaboración y la lectura del manuscrito. 